\title{
Anaesthetic, Procedure and Complications Management of Serial Whole-Lung Lavage in an Obese Patient with Pulmonary Alveolar Proteinosis: Case Report
}

\author{
Helena Marta Rebelo ${ }^{1}$, Luisa Guedes ${ }^{1}$, Dalila Veiga ${ }^{1}$, Antonio C. Fiuza ${ }^{1}$, Fernando Abelha ${ }^{2}$
}

\begin{abstract}
Summary: Rebelo H, Guedes L, Veiga D, Fiuza A, Abelha F - Anaesthetic, Procedure and Complications Management of Serial Whole Lung Lavage in an Obese Patient with Pulmonary Alveolar Proteinosis: Case Report.

Background and objectives: The first case of Pulmonary Alveolar Proteinosis (PAP) was described by Rose in 1958, but it is still a rare disorder. PAP is characterized by deposition of lipoproteinaceous material secondary to abnormal processing of surfactant by macrophages. Patients may suffer from progressive dyspnea and cough that at times is accompanied by worsening hypoxia and its course can vary from progressive deterioration to spontaneous improvement. Many therapies have been used to treat PAP including antibiotics, postural drainage, and intermittent positive pressure breathing with aerosolized Acetylcysteine, heparin and saline. At present, the mainstay of treatment is whole lung lavage (WLL). Although generally well tolerated, WLL can be associated with some complications.
\end{abstract}

Case Report: We report a case of severe PAP through the anaesthetic, procedure and complications management of pulmonary alveolar proteinosis in one patient who has undergone multiple, alternating, single-lung lavages over the past seven years, the last three in our hospital, with improvements in her symptoms following each therapy.

Keywords: Anesthesia, General; Broncoalveolar Lavage; Intraoperative Complications; Pulmonary Alveolar Proteinosis.

@2012 Elsevier Editora Ltda. All rights reserved.

\section{INTRODUCTION}

The first case of Pulmonary Alveolar Proteinosis (PAP) was described by Rose in $1958^{1-4}$ but it remains a rare disorder. PAP is characterized by deposition of lipoproteinaceous material secondary to abnormal processing of surfactant by macrophages $2,3,5-11$.

Patients may suffer from progressive dyspnea and cough; at times accompanied by worsening hypoxia ${ }^{3,12}$; its course can vary from progressive deterioration to spontaneous improvement ${ }^{1-3}$.

Many therapies have been used to treat PAP over the years, including antibiotics, postural drainage, and intermittent positive pressure breathing with aerosolized Acetylcysteine, heparin and saline ${ }^{13}$.

At present, the mainstay of treatment is whole lung lavage (WLL) ${ }^{3,6,8,9,12,13}$, first described for Ramirez-Rivera et al. ${ }^{6,9,14,15}$

Received from Anesthesiology Service, Hospital São João, Brazil.

1. MD, Anesthesiology Service, Hospital São João, Brazil

2. PhD, Anesthesiology Service, Hospital São João, Brazil

Submitted on December 2, 2011.

Approved on February 22, 2012.

Correspondence to:

Helena Marta Rebelo, MD

Hospital São João

Alameda Prof. Hernâni Monteiro

4200-319- Porto, Portugal

E-mail: helena.rebelo.net@gmail.com
There are three variants of the disease: congenital, secondary and acquired 2,16. Recent insights suggest that, in the most common form, acquired (idiopathic) PAP, autoimmunity against pulmonary granulocyte- macrophage colonystimulating factor (GM-CSF) plays a major role $2,3,6,8,10-12,14$. Recombinant Granulocyte- Macrophage colony stimulating factor appears to benefit a subset of adult patient with PAP and may represent an alternative in the treatment of this disease $8,10,11,14$, but the success rate is not yet sufficient to displace WLL ${ }^{16}$.

Although generally well tolerated, WLL can be associated with some complications ${ }^{17}$.

We report a case of severe PAP throughout the anesthetic procedure and complications' management in one patient who has undergone multiple, alternating, single-lung lavages over the past seven years, the last three in our hospital, with improvements in her symptoms following each therapy.

\section{Case Report}

We describe a case of PAP in a 50-year-old black women, obese $(\mathrm{BMI}=41.5)$ and hypertensive, controlled with nifedipine. Seven years before admission, she had been diagnosed with primary PAP after investigation of complaints of exertional dyspnea, cough and mucous sputum for five months. The diagnosis was based on compatible imaging studies (chest tomography showing interstitial infiltrates and "crazy paving" aspect) and characteristic milky bronchoalveolar-lavage (BAL) fluid with granular material staining with periodic acid-Schiff. 
Respiratory function test showed a forced expiratory volume in the first second (FEV1) of $1.17 \mathrm{~L}$ (57\% of predicted), a Tiffeneau index (IT) of $65 \%$ and total lung capacity (TLC) of $3.31 \mathrm{~L}$ (63\% of predicted). Diffusing capacity (DLCO) was $35 \%$ of predicted. A right whole-lung BAL was performed in May 2004 at another hospital, when symptoms and arterial blood gas (ABG) parameters worsened.

In January 2011, because of the patient's worsening symptoms, she was admitted in Hospital São João with clinical and functional worsening: five week progressive exertional dyspnea, nocturnal dyspnea, mild cough with clear sputum. She presented other medical problems - such as obesity and hypertension - and had no history of weight loss, hemoptysis or night sweats and no significant family or occupational history.

On examination at admission, the patient was hypoxemic: her chest was clear but with diminished breath sounds, with no peripheral edema, with normal arterial pressure and heart rate. While breathing room air at rest, the gas blood sample showed arterial hemoglobin oxygen saturation $\mathrm{SaO}_{2}$ 86,7\%; arterial partial pressure of oxygen $\mathrm{PaO}_{2} 49.9 \mathrm{~mm} \mathrm{Hg}$ and with a moderate restrictive ventilatory defect (respiratory pulmonary function on 5/1/2011: forced vital capacity $(F V C)=1.22 \mathrm{~L}(36.6 \%)$; FEV1 = 1.20 L (42.1\%); IT = 98.86\%; TLC = 2.81 L (52.4\%); residual volume $(\mathrm{RV})=1.40 \mathrm{~L}(76.2 \%) ; \mathrm{RV} / \mathrm{TLC}=140 \%$; functional residual capacity $(\mathrm{FRC})=2.81 \mathrm{~L}(80 \%)$; $\mathrm{DLCO}=5.5 \%$; DLCO/VA (alveolar volume) $=(18.6 \%)$.

The chest X-ray showed bilateral patchy air-space infiltration, the Thoracic Computed Tomography indicated bilateral ground glass infiltrate (with no lateralization), with interlobular septal thickening, increase in the diameter of the pulmonary artery trunk suggesting pulmonary hypertension.

Due to patient's worsening symptoms, bronchoalveolar lavage was performed. Lung lavage was carried out on 3 occasions over the last year. Each time, the patients experienced an improvement in her symptoms and chest radiographic findings.

After preoxygenation for 5 minutes, anesthesia was induced with propofol, fentanyl and subsequently maintained with sevoflurane and remifentanyl infusion. Rocuronium was administered to facilitated tracheal intubation and during the maintenance of anesthesia. Lung separation was achieved by placing a 37F left double-lumen endobronchial tube into the left mainstem bronchus and its correct position confirmed with auscultation and fibrotic bronchoscopy. Radial arterial and jugular venous catheters were inserted to maintain continuous hemodynamic monitoring. Ventilator parameters were adjusted to patient along the WLL. Pressure control ventilation with minimum positive end expiratory pressure (PEEP) values of $6 \mathrm{mmHg}$ and a plateau pressure under $30 \mathrm{~mm} \mathrm{Hg}$ was set. Throughout the procedure invasive blood pressure, central venous pressure (CVP), urine output, $\mathrm{SatO}_{2}$, capnography and esophageal temperature were continuously monitored and repeated $A B G$ s were made.

The patient's temperature was maintained using lavage fluid warmed to body temperature, warming blanket and intravenous fluid warmer.

Lavage was performed with $1,000 \mathrm{~mL}$ of normal saline warmed to body temperature with the patient in prone posi- tion (30 degree angle anti-Trendelemburg). The inflow of fluid was made by gravity from a height about $30 \mathrm{~cm}$ above the midaxillary line.

Chest percussions were performed for some minutes while fluid was indwelling to help loosen proteinacious material. The fluid was then gravitationally drained and sampled. This process was repeated until the effluent cleared or some complication emerged. After the first procedure we choose to use a CPAP balloon ventilation with $10 \mathrm{~mm} \mathrm{Hg}$ pressure limit and abandoned chest percussions. This balloon expanded the flooded lung after half of the fluid was washout.

During fluid inflow and outflow, airway pressure and tidal volume were closely monitored to assess for leakage of fluid from the non-ventilated lung into the ventilated lung.

The potential electrolyte disorders due to the effect of dialysis on the pulmonary lavage (mainly, hypokalemia and metabolic acidosis) were compensated using appropriate therapy.

At the end of the procedure, two-lung ventilation was started and recruitment maneuvres were applied to restore expansion of both lungs. After every WLL procedure, endotracheal tube was exchanged, patient was transferred for postanesthethic care unit and the trachea was extubated the next morning. This was done in the hope that after this time span the excess fluid in the lungs had been absorbed or suctioned and a good chest toilet and overnight ventilation had been done.

A similar anaesthetic technique was used for each wholelung lavage procedure.

In the first WLL, the procedure was interrupted because there was a spillage of lavage fluid into the ventilated lung with bronchospasm and difficulty in keeping ventilation in a physiologic range. The patient developed respiratory acidosis and hypoxia and fluid was suctioned with bronchoalveolar fibroscopy. This has led to the interruption of the procedure and the beginning of two-lung ventilation. Additionally, inhalation of salbutamol plus ipratropium bromide and intravenous aminophylline were administered.

During the subsequent WLL, salbutamol was administered by continuous intravenous infusion in order to prevent lavageinduced bronchospasm, which may have reduced the efficacy of the procedure.

At the third WLL, after the turnover of a total volume of $4 \mathrm{~L}$, the progressive flooding of the non-ventilated lung resulted in airway mechanical compression leading to respiratory acidosis and hypoxia imposing the end of the procedure.

In the last two lung lavages, it was necessary to use noradrenaline infusion as a vasopressor to sustain blood pressure, but it was discontinued during the same day.

Despite the complications that occurred, post lavage radiographs showed partial clearing of PAP and pulmonary mechanics and gas exchange improved.

\section{DISCUSSION}

Pulmonary alveolar proteinosis is an interesting rare disease, which was first described recently, in $1958^{3}$. The affected individuals suffer a progressive accumulation of lipoproteins in 
the alveolar space $2,3,6-8,11,12,18$ as a result of defective macrophage clearance $2,8,11,12,14$, yet with minimal local lung inflammation or distortion of architecture on biopsy ${ }^{3,9,12}$. Recent insights into the pathogenesis of PAP suggest that in most cases of PAP autoimmunity against pulmonary granulocytemacrophage colony-stimulating factor (GM-CSF) plays a major role 2,6,8,9,11,12,14,18.

This condition is characterized by worsening of gas exchanges leading to progressive dyspnea, cough and hypoxemia in patients in their third and fourth decades $2,6,8,12$.

Most patients are male $(70 \%)^{2,6,8,9,12}$, and the majority have a significant smoking history ${ }^{6,12}$. Clinical presentation is variable and non-specific ${ }^{2}$, often leading to months or years of misdiagnosis ${ }^{11,13}$.

Three variants of the disease have been recognized: congenital, secondary and acquired (or idiopathic) ${ }^{2,16}$. The later is the one of most importance concerning prevalence (approximately 0.2 cases per million of population) and response to treatment $8,9,14$. Investigations reveal radiographic bilateral patchy air-space infiltration 6,9,11,12,14, restrictive pulmonary function 5,6,12,17, impaired diffusion capacity ${ }^{12}$ and milky brochoalveolar lavage (BAL) fluid-rich in alveolar macrophages ${ }^{6,11,16}$

Although increasing evidence indicates that GM-CSF therapy may be beneficial for patients with PAP 3,9-11,14,18, the success rate for GM-CSF is so far not sufficient to displace the success of WLL therapy ${ }^{16}$. In some studies, approximately $50-70 \%$ of patients following GM CSF treatment protocols have obtained significant improvement in symptoms, physiological and radiological findings, which mean that a significant percentage failed to benefit ${ }^{8}$.

Apart from the failure rate, the inconvenience of daily injections and the costs associated with the procedure should also be considered. Recently, concerns about the security involved in the supply of this treatment have to be taken into account. In addition, the potential toxicity of GM CSF long term use is not yet fully understood ${ }^{8}$.

The mainstay of treatment is still bronchopulmonary lavage, introduced by Ramirez in late 1960 6,8,12,16,18.

It is usually indicated only in PAP patients with significant symptoms and hypoxemia 7,9,14,16. The clinical and physiologic response to WLL has been attributed to the removal of lipoproteinaceous material from the alveolar space ${ }^{7,12}$, but additional mechanisms including the bulk removal of anti- GMCSF antibody as well as other possible immunologic effects on the effectors cells, such as alveolar macrophage or type II epithelial cell are possible ${ }^{6,9}$. Almost all patients respond to WLL, with subjective improvement, ranging from few months to few years ${ }^{19}$.

The physiologic improvement includes increases in forced vital capacity, increased total lung capacity, a higher $\mathrm{PaO}_{2}$ at rest and with exercise, and a better diffusing capacity of the lungs for carbon monoxide, with decreases in the alveolararterial gradient and shunt fraction ${ }^{13}$. Other noted signs of improvement have been described as gradual radiographic clearing, decreased LDH levels, increased ventilation, perfusion, and ventilation/perfusion matching on scintigraphy ${ }^{13}$.
Anesthesia for WLL is undoubtedly hazardous: the use of one lung ventilation and instillation of large volumes of fluid in the setting of pre-existing respiratory failure put the patient at risk of profound hypoxemia ${ }^{6}$. In the patient here described, there was the added difficulty in ventilation associated to morbid obesity. Obese patients have unique characteristics, with cardiovascular and pulmonary changes requiring ventilation to be individualized ${ }^{20}$. Other particularities may come from the presence of other comorbidities; necessarily difficult venous accesses and positioning are expected difficulties ${ }^{20}$.

In WLL under general anesthesia, the two lungs are separated by endobronchial intubation and one lung is ventilated while the other is flooded with warm saline to progressively wash out the alveolar space 8,9,16,17. Although there have been several refinements in this technique and developments in anesthesia and perioperative care, the basis of the treatment remains the same ${ }^{8}$.

The use of a right sided tube should be avoided, as it tends to block the orifice of the right upper lobe bronchus. Additionally, the shape of the cuff and the presence of right upper lobe ventilation slit make an airtight seal difficult to achieve ${ }^{6}$. The lungs should be functionally separated at a pressure of $50 \mathrm{~mm} \mathrm{Hg}$ to prevent leakage of lavage fluids around the cuff ${ }^{19}$.

Preoxygenation for five minutes before induction is extremely important because failure of denitrogenation of lungs leaves nitrogen bubbles in alveoli and limits the effectiveness of lavage $14,16,19$.

This is generally well tolerated ${ }^{17}$ but there are some potential complications. The major one is intraoperative refractoriness, which tends to be more common while the first lung is being lavaged. Low oxygen saturations (percentages of 70 s to 80s) are not uncommon early in the procedure; however, they generally improve throughout the procedure without any intervention ${ }^{17}$. The hypoxemia is related to ventilation-perfusion mismatch during lung lavage: during the filling phase, the perfusion of the non- ventilated lung is reduced by compression of the pulmonary vasculature and, hence, the shunt is reduced; however, during the drainage phase, reperfusion of the nonventilated lung increases shunt causing hypoxemia 5,6,16.

Although the use of PEEP applied to the ventilated lung during the drainage phase may augment the shunt ${ }^{5,6}$ we advocated its use because during the filling phase of the nonventilated lung, the ventilated lung may improve oxygenation. Besides that, in the obese a larger safety margin could be obtained by using PEEP due to recruitment of alveolis. Thus, this use would allow smaller inspired oxygen fractions, which is desirable regarding the intra and postoperative prevention of atelectasis ${ }^{20,21}$.

Several other strategies have been suggested for management of hypoxemia, including manual ventilation of partially fluid-filled lung 6,22, intermittent double-lung ventilation 6,23, concomitant use of inhaled nitric oxide ${ }^{6,24}$, and ipsilateral pulmonary artery occlusion of the non-ventilated lung using 
pulmonary artery catheter 6,25 . Hyperbaric oxygen and venovenous extracorporeal membrane oxygenation have been reported to be useful 6,17 , but in more recent studies they have not been found necessary in most cases ${ }^{17}$.

Other complications include pneumothorax, pleural effusion, and hydropneumothorax, which can be avoided by meticulous charting of the infused saline solution and the output, and by taking care not to allow instilled fluid to exceed the fluid drained by more than a few hundred milliliters in consecutive lavages ${ }^{17}$.

Infusion of large volumes of saline in lungs is associated with mediastinal shift, increased intrathoracic pressure, increased CVP, higher arterial oxygen tension and hypotension ${ }^{14}$.

We report a mechanical difficulty in ventilation, probably due to the lower pulmonary compliance worsening by the infusion of fluid in non-ventilated lung and due to morbid obesity which may have caused a diminished compliance in the chest wall.

An associated problem that is described during the procedure is the displacement of the endotracheal tube. It can lead to a leak through the double lumen tube (DLT) resulting in spillage of lavage fluid into the ventilated lung ${ }^{14,16}$, so it is mandatory to confirm the placement of DLT by fiberoptic bronchoscope.

It was performed WLL using isotonic saline infused by gravity from a height of $40 \mathrm{~cm}$ above the midaxillary line. The resultant hydrostatic infusion pressure of lavage fluid with this setup is $30 \mathrm{~mm} \mathrm{Hg}{ }^{19}$, became a method of controlling the pressure exerted in the endobroncheal tube cuff. With this procedure we tried to prevent an exceeding of the cuff pressure preventing a leakage and displacement.

Monitoring of airway pressure and tidal volume during one lung ventilation is crucial to detect fluid leakage into the ventilated lung ${ }^{6}$.

An increase in airway pressure or a decrease in tidal volume may indicate a reduction in compliance of the ventilated lung and fluid leakage should be considered. Aside from that, monitoring to detect the loss of lung isolation can be done by:

i. The appearance of bubbles in the lavage fluid draining from the lavaged side.

ii. Appearance of rales and rhonchus on the ventilated side.

iii. Imbalance between the instilled and the drained volumes 6,17 .

Fiberoptic bronchoscopy inspection will confirm if flooding of the ventilated lung has occurred.

Traditionally, the procedure is conducted in lateral decubitus to lavage dependent lung and ventilating the nondependent lung to minimize the risk of spillage of saline to the other lung 6,19. This is however associated with higher ventilation perfusion mismatch in an already severely hypoxic patient 14,17,19. The lateral decubitus position with lavaged lung nondependent has the advantage to minimize blood flow to the nonventilated lung, but maximizes the possibility of spillage ${ }^{19}$.

Like some other reports ${ }^{17}$, because of the difficulties associated with the obesity, it was chosen to perform the procedure in supine position but with a $30^{\circ}$ anti-Trendelemburg not only to minimize the risk of flood the ventilated lung but also because that positioning is favorable in obese patients.

To these patients, supine position may lead to cardiorespiratory instability ${ }^{20,21}$. That position increases the pressure of the intestines on the diaphragm, hindering ventilation, leading to hypoxemia and hypercapnia; it increases cardiac output and the pressure in the pulmonary artery ${ }^{21}$. The left lateral decubitus may be used to decrease the compression of the inferior vena cava. The semi-sitting position associated with the lateral decubitus has a lower degree of cardiorespiratory compromise. A $30^{\circ}$ to $45^{\circ}$ elevation of the thorax improves cardiorespiratory function and increases the safe period of apnea ${ }^{21}$.

Treatment of flooded lung involves rapid endobronchial suctioning followed by effective re-expansion of the flooded lung and readjustment of double lumen endotracheal tube to ensure no further ongoing leak ${ }^{6,17}$. Patient positioning should be carried out carefully in order to avoid endotracheal tube movement.

Although manual chest percussion associated with positional changes during WLL seems to enhance material recovery ${ }^{18}$, this procedure was used only in the first WLL, because it may be associated to a higher risk of displacement of the endotracheal tube. We adopted the "Bingisser modification" ${ }^{18}$, that consists in several cycles of manual ventilation using a CPAP valve with $10 \mathrm{~mm} \mathrm{Hg}$ pressure limit, after recovering half of the amount of instilled water ${ }^{18}$. That technique improves the removal of the accumulated material ${ }^{18}$ and permits control of exerted pressure.

During the procedure, all measures should be taken to avoid hypothermia since the procedure takes several hours to complete and is done with lavage fluid at room temperature ${ }^{14}$.

Temperature monitoring is essential and could be maintained using lavage fluid warmed to body temperature, warming blanket and intravenous fluid warmer ${ }^{14}$.

In the case of lateral decubitus and because the procedure can be time consuming, meticulous care need to be taken to avoid ischemia complications to the extremities by placing supporting pillows in the dependent axilla, under the head and between the thighs ${ }^{17}$.

Though post lavage, radiographs show partial clearing of PAP compared with baseline pre-wash radiograph, pulmonary mechanics and gas exchange improve after the lavage.

We described an acceptable anesthetic technique for WLL in a morbidity obese patient with severe respiratory failure due to PAP and discussed the associated complications and its management. 


\section{REFERENCES}

1. Ang BS, Poh WT, Ong YY, Chiang GS - Pulmonary alveolar proteinosis: a case report. Singapore Med J, 1991;32:454-456.

2. Trapnell BC, Whitsett JA, Nakata $\mathrm{K}$ - Pulmonary alveolar proteinosis. N Engl J Med, 2003;349:2527-2539.

3. Seymour JF, Presneill JJ - State of the art-pulmonary alveolar proteinosis progress in the first 44 years. Am J Resp Crit Care Med, 2002;166:215-235.

4. Rosen $\mathrm{SH}$, Castleman B, Liebow AA - Pulmonary alveolar proteinosis. N Engl J Med, 1958;258(23):1123-1142.

5. Hodges $\mathrm{O}$, Zar HJ, Mamathuba R, Thomas $\mathrm{J}$ - Bilateral partial lung lavage in a obese patient with pulmonary alveolar proteinosis. $\mathrm{Br} \mathrm{J}$ Anaesth, 2010;104(2):228-230.

6. Stephen TW, Evans JA - Anaesthesia for serial whole-lung lavage in a patient with severe pulmonary alveolar proteinosis: a case report. $J$ Med Case Rep, 2008;2:360.

7. Perez A $4^{\text {th }}$, Rogers RM - Enhanced alveolar clearance with chest percussion therapy and positional changes during whole-lung lavage for alveolar proteinosis. Chest, 2004;125(6):2351-2356.

8. Morgan $\mathrm{C}-$ The benefits of whole lung lavage in pulmonary alveolar proteinosis. ERJ, 2004;23(4):503-505.

9. Kavuru MS, Popovich $\mathrm{M}$ - Therapeutic whole lung lavage: a stop-gap therapy for alveolar proteinosis. Chest, 2002;122(4):1123-1124.

10. Greenhill SR, Kotton DN - Pulmonary alveolar proteinosis: a benchto-bedside story of granulocyte-macrophage colony-stimulating factor dysfunction. Chest, 2009;136(2):571-577.

11. Borie R, Danel C, Debray MP, Taille C, Dombret MC, Aubier M, Epaudf R, Crestani B - Pulmonary alveolar proteinosis. European Respiratory review, 2011;20(120):98-107.

12. Simpson RI, Ramsay MA, Millard MA, Capehart JE - Management of pulmonary alveolar proteinosis by repeated bronchoalveolar lavage. Proc (Bayl Univ Med Cent), 2000;13:119-120.

13. Mazone $P$, Thomassen MJ, Kavuru M - Our new understanding of pulmonary alveolar proteinosis: what an internist needs to know. Cleve Clin J Med, 2001;68(12):977-978..

14. Nandkumar S, Butani M, Udwadia Z - Pulmonary alveolar proteinosis with respiratory failure-anaesthetic management of whole lung lavage. Indian J Anaesth, 2009;53(3):362-366.

15. Dixit R, Chaudhari LS, Mahashur AA - Anaesthetic management of bilateral alveolar proteinosis for bronchopulmonary lavage. J Postgrad Med, 1998;44(1):21-23.

16. Aguiar M, Monteiro $P$, Marques $M M$ et al. - Lavagem pulmonar total - a propósito de quatro casos de proteinose alveolar. Rev Port Pneumol, 2009;15(1).
17. Michaud G, Reddy C, Ernst A - Whole-lung lavage for pulmonary alveolar proteinosis. Chest, 2009;136(6):1678-1681.

18. Luisetti M, Kadija Z, Mariani F, Rodi G, Campo I, Trapnell BC - Therapy options in pulmonary alveolar proteinosis. Ther Adv Respir Dis, 2010;4:239-248.

19. Rogers MC (ed) - Principles and practice of anesthesiology. Mosby Year Book, 1993, pp. 1824-1829.

20. Oakes DD, Cohn RB, Brodsky JB, Merrell RC, Sherck JP - Obesity lateral thoracotomy and one-lung anesthesia in patients with morbid. Ann Thorac Surg, 1982;34:572-580.

21. Lorentz MN, Albergaria VF, Lima FAZ - Anestesia para obesidade mórbida. Rev Bras Anestesio, 2007;57(2):199-213.

22. Bingisser R, Kaplan V, Zollinger A, Russi EW - Whole-lung lavage in alveolar proteinosis by a modified lavage technique. Chest, 1998;113(6):1718-1719.

23. Ahmed $\mathrm{R}$, Iqbal $\mathrm{M}$, Kashef $\mathrm{SH}$, Almomatten $\mathrm{MI}$ - Whole lung lavage with intermittent double lung ventilation. A modified technique for managing pulmonary alveolar proteinosis. Saudi Med J, 2005;26(1):139141.

24. Moutafis M, Dalibon N, Colchen A, Fischler M - Improving oxygenation during bronchopulmonary lavage using nitric oxide inhalation and almitrine infusion. Anest Analg, 1999;89(2):302.

25. Nadeau MJ, Côté D, Bussières JS - The combination of inhaled nitric oxide and pulmonary artery balloon inflation improves oxygenation during whole-lung lavage. Anesth Analg, 2004;99(3):676-679. 